\title{
Microbiology Risk Factors and Outcomes of Peritonitis in Tunisian Peritoneal Dialysis Patients
}

\author{
Lilia Ben Lasfar, ${ }^{\mathrm{a}}$, Yosra Guedria ${ }^{\mathrm{a}}$, Awatef Azzebia ${ }^{\mathrm{a}}$, Wissal Sahtout ${ }^{\mathrm{a}}$, Sinda Mrabet ${ }^{\mathrm{a}}$, \\ Asma Fradia , Salma Toumi ${ }^{\text {a }}$, Ferdaous Sabria, Samira Ben Amor ${ }^{\mathrm{a}}$, \\ Dorsaf Zallema ${ }^{a}$, Abdellatif Achour ${ }^{\mathrm{a}}$
}

\begin{abstract}
Background: Peritonitis is a major complication of peritoneal dialysis (PD), accounting for considerable mortality and hospitalization among PD patients. We have performed a single unit study examining rate of peritonitis, causative organisms, clinical outcomes and impact on technique failure and patient survival.
\end{abstract}

Methods: It was a retrospective review of the medical records of 182 PD patients who were followed up from January 2006 through June 2016. We have listed 186 episodes of peritonitis.

Results: The overall incidence of peritonitis during the 10-year study period was one episode every 27.25 month-patient. The mean time to first peritonitis after beginning PD was $14.25 \pm 16$ months $(0-65)$. Gram-positive organisms were the main implicated agents and caused $27.92 \%$ of peritonitis. In multivariate Cox regression, no correlation was associated with diabetes, cardiovascular disease and residual renal function at baseline. In the Kaplan-Meier analysis, the peritonitis group was not correlated with more loss of residual renal function. More dropouts from PD were observed compared to the peritonitisfree group $(\mathrm{P}<0.000)$, but no influence with patient survival.

Conclusion: Gram-positive organisms were the main causative agents. And peritonitis was associated to technique failure but not with patient survival.

Keywords: Peritonitis; Risk factor; Technique survival; Patient survival

\section{Introduction}

Peritonitis is the most common complication of peritoneal di-

\footnotetext{
Manuscript submitted May 22, 2018, accepted June 18, 2018

aDepartment of Nephrology, Dialysis and Kidney Transplantation, Sahloul Hospital, Sousse, Tunisia

${ }^{\mathrm{b}}$ Corresponding Author: Lilia Ben Lasfar, Department of Nephrology, Dialysis and Kidney Transplantation, Sahloul Hospital, Sousse, Tunisia.

Email: lililasfar@gmail.com
}

doi: https://doi.org/10.14740/wjnu349w alysis (PD) patients. Despite reductions in peritonitis rates, it remains the major leading cause to technique failure among end-stage renal disease (ESRD) undergoing PD and so switch to hemodialysis (HD) $[1,2]$.

The majority of peritonitis cases are caused by pathogenic bacteria (in most cases, Gram-positive cases), with a small number of cases caused by fungi, mostly of the Candida species [3].

Sources of bacterial peritonitis include intra-luminal by touch contamination, periluminal (catheter-related infection), transvisceral migration due to intra-abdominal pathology and rarely hematogenous spread.

Peritonitis is a serious complication of PD and is responsible of a significant morbidity and mortality [4]. It causes $30 \%$ of technique failures and $21 \%$ of infectious deaths in Australian and New Zealand PD patients [3].

This study reports the rate, causative organisms and impact of peritonitis on technique failure and patient survival of all episodes of PD-associated peritonitis in a Tunisian PD unit.

According to the second edition of the Renal Association Standards Document [5], peritonitis rates should be less than one episode per 18 months, and the negative peritoneal fluid culture rate in patients with clinical peritonitis should be $<$ $10 \%$.

\section{Patients and Methods}

All patients starting continuous ambulatory peritoneal dialysis (CAPD) or automated peritoneal dialysis (APD) at Sahloul Hospital in Sousse, Tunisia between January 2006 and June 2016 were followed up. The empiric antibiotics used in the unit after the initial diagnosis of peritonitis were an aminoglycoside and vancomycin. No patients continued on APD during episodes of peritonitis.

PD-associated peritonitis was defined if the peritoneal effluent contained $>100$ white cells $/ \mu \mathrm{L}$ with $>50 \%$ polynuclear leukocytes.

The operating procedure for culture of PD fluid was obtained from the microbiology departments.

Peritonitis rate was calculated as the number of infections for all patients divided by the number of patient-months on PD.

All patients were followed till death, renal transplant, 
Table 1. Clinical Characteristics of Patients

\begin{tabular}{|ll}
\hline Patients (n) & 182 \\
Sex (male/female) & $109: 73$ \\
Age (years, mean \pm standard deviation) & $43.93 \pm 16.95$ \\
Mean duration of follow-up (months) & $27.75 \pm 26.18$ \\
Underlying disease, $\mathrm{n}(\%)$ & \\
$\quad$ Chronic glomerulonephritis & $25(13.73)$ \\
$\quad$ Diabetes mellitus & $64(35.16)$ \\
$\quad$ Hypertension & $16(8.79)$ \\
\multicolumn{1}{l}{ Interstitial nephropathy } & $41(22.52)$ \\
$\quad$ Others or unknown & $36(19.8)$ \\
\hline
\end{tabular}

switch to HD or the end of the study on June 2016.

The data collected included at baseline (the start of dialysis therapy): 1) demographic and clinical data: age, sex, presence of diabetes, hypertension, comorbid conditions, residual renal function (RRF); 2) biochemical data: levels of hemoglobin, blood albumin, cholesterol, calcium, phosphorus, ferritinemia, C-reactive protein, protein catabolic rate (NPCR); and 3) ultrafiltration, types of antihypertensives, types of dialysate.

Microbiological characteristics were identified for each episode of peritonitis.

\section{Clinical outcomes}

Outcomes were represented by residual renal function, technique and patient survival.

Loss of RRF was defined as an RRF inferior or equal to $2 \mathrm{~mL} / \mathrm{min}$.

Technique survival was defined as the persistence of the technique use until permanent transfer to HD due to inadequate dialysis, peritonitis, ultrafiltration failure and mechanical or operational problems. For the analysis, the end-point event was transfer to $\mathrm{HD}$, whereas transplantation or death on $\mathrm{PD}$ was the censored observation.

Patient survival was defined as the probability of patients surviving on PD. The analysis of patient survival from any cause, death was the end-point event, while switch to HD or transplantation was the censored observation.

\section{Statistical analysis}

The data were expressed as mean $\pm \mathrm{SD}$ for continuous variables and numbers (n) and percentages (\%) for categorical variables.

For univariate analysis of the continuous variables, we used the ANOVA test and for univariate analysis of the categorical variables, we used the Chi-square test.

The analyzed variables included sex, age, anuria, ultrafiltration, comorbidities, biochemical data and baseline PD adequacy.

On the other hand, the multivariate analysis was performed with the Cox regression model to identify the statisti-
Table 2. Distribution of Patients According to the Number of Peritonitis

\begin{tabular}{|ll|}
\hline Number of peritonitis & Number of patients \\
\hline 1 & 46 \\
\hline 2 & 21 \\
\hline 3 & 14 \\
4 & 5 \\
\hline 5 & 3 \\
\hline 6 & 2 \\
9 & 1 \\
\hline
\end{tabular}

cally significant risk factors associated with peritonitis.

To investigate the association of peritonitis with patients' outcomes, RRF loss, curves of technique and patient survival were performed with the Kaplan-Meier model.

A P value inferior to 0.05 was considered statistically significant.

\section{Results}

\section{Demographic and clinical characteristics of patients}

A total of 182 patients with ESRD started CAPD or APD during the 10-year period. The mean age of the incidents patients was $43.93 \pm 16.95$ years and $59.9 \%$ were males. The average duration of follow-up is $27.75 \pm 26.18$ months.

One hundred and seven patients had a follow-up duration of more than 2 years and 36 patients had a follow-up duration of more than 4 years.

The baseline characteristics of 182 patients are shown in Table 1.

\section{Peritonitis rate, microbiological characteristics and con- tamination pathways of the first peritonitis}

\section{Peritonitis rate}

The overall incidence of peritonitis during the 10-year study period was 0.44 episodes per patient-year which equals one episode every 27.25 month-patient. The mean time to first peritonitis after beginning PD was $14.25 \pm 16$ months $(0-65)$.

One hundred and eighty-six episodes of peritonitis occurred over the total study period (54 episodes during the first year). The peritonitis-free group represents $48.9 \%$ of the total population $(n=89)$. Peritonitis occurred in $51.1 \%$ of cases $(1$ -9 episodes were noted per patient).

Other patients had 1 - 9 peritonitis (Table 2).

\section{Causative organisms of peritonitis}

A total of 186 episodes of peritonitis were recorded during the 
Table 3. Details of Causative Organisms of the First Peritonitis From 2006 to 2016

\begin{tabular}{|cl|}
\hline Group of microorganism & $\mathbf{n}(\mathbf{\%})$ \\
\hline Gram-positive cocci & $26(27.92)$ \\
\hline Staphylococcus aureus & $16(17.20)$ \\
\hline Staphylococcus epidermidis & $3(3.22)$ \\
\hline Streptococcus & $7(7.5)$ \\
\hline Gram-negative rods & $22(23.9)$ \\
\hline Pseudomonas & $6(6.45)$ \\
Acinetobacter & $2(2.15)$ \\
Enterobacter & $3(3.22)$ \\
Klebsiella pneumoniae & $5(5.37)$ \\
Escherichia coli & $6(6.45)$ \\
\hline Candida albicans & $5(5.37)$ \\
\hline Aspergillus & $1(1.07)$ \\
\hline Others & $4(4.3)$ \\
\hline Culture-negative & $34(36.55)$ \\
\hline
\end{tabular}

study. The causative organisms of peritonitis are listed in Table 3.

Gram-positive organisms caused 26 episodes of peritonitis $(27.92 \%)$, Gram-negative organisms were identified in 22 episodes $(23.9 \%)$, fungi in six episodes $(6.5 \%)$, and no case of $M$. tuberculosis has been reported.

There were 34 episodes $(36.55 \%)$ of culture-negative peritonitis. In culture-positive episodes, the most cases $(44.82 \%)$ were caused by a single Gram-positive organism: $17.2 \%$ by S. aureus, $3.22 \%$ by $S$. epidermidis, and $7.5 \%$ by Streptococcus. Single Gram-negative organisms accounted for $37.9 \%$ of culture-positive peritonitis and were mainly caused by $E$. coli and Pseudomonas (Table 3): Pseudomonas in 6.45\%, Acinetobacter in $2.15 \%$, Klebsiella in $5.37 \%$, Serratia in $1.3 \%$, and Enterobacter in $3.22 \%$.

\section{Contamination pathways}

The causes of peritonitis were varied but the main cause was the lack of asepsis (Table 4).

\section{Analysis of risk factors for peritonitis}

Patients were classified as peritonitis-free $(n=89)$ and peritonitis group $(\mathrm{n}=93)$. Table 5 shows the differences between the two groups. In the peritonitis group, diabetes, hypertension, and cardiovascular disease were more prevalent but the difference was not significant. Levels of ultrafiltration, CRP and ferritin were higher also, in this group.

In multivariate Cox regression, no correlation was associated with diabetes, cardiovascular disease and RRF at baseline (Table 6).
Table 4. Causes of Peritonitis

\begin{tabular}{lll}
\hline & Number & Percentage \\
\hline Lack of asepsis & 140 & 78.2 \\
Extender crack & 2 & 1.1 \\
Endogenous cause & 2 & 1.1 \\
Other infectious source & 4 & 2.2 \\
Exit-site infection & 14 & 7.8 \\
Catheter disconnection & 3 & 1.6 \\
Catheter breaking & 1 & 0.5 \\
Catheter crack & 2 & 1.1 \\
Defective material & 4 & 2.2 \\
Unknown cause & 7 & 3.9 \\
\hline
\end{tabular}

\section{Clinical outcomes}

\section{$R R F$}

In the Kaplan-Meier analysis, the peritonitis group was not correlated with more loss of RRF (Fig. 1).

\section{Technique survival}

In Kaplan-Meier analysis, the peritonitis group was significantly correlated with more dropouts from PD compared to the peritonitis-free group $(\mathrm{P}<0.000)$ (Fig. 2).

\section{Patient survival}

Twenty-one patients died among the total population. Among deaths, only three cases were related to a septic cause.

In the Kaplan-Meier analysis, there was no difference in patient survival between peritonitis group and peritonitis-free group (Fig. 3).

\section{Discussion}

This study tries to summarize the peritonitis situation: incidence, risk factors and impact on technique and patient survival in our unity of PD.

The overall peritonitis rate was one episode every 27.25 month-patient. We converted the incidence to patient-month to compare with last studies.

In Scotland, in a study including 10 adult renal units and 1,205 patients, the overall peritonitis rate was one every 19.2 month-patient (between 1999 and 2002) which is a superior incidence compared with our study. In fact, the variability of results is due to the variability of the sample size. The Renal Association Standard recommends one episode every 18 months [1].

Although the incidence of peritonitis varies from unit to 
Table 5. Comparison Between Peritonitis-Free and Peritonitis Group

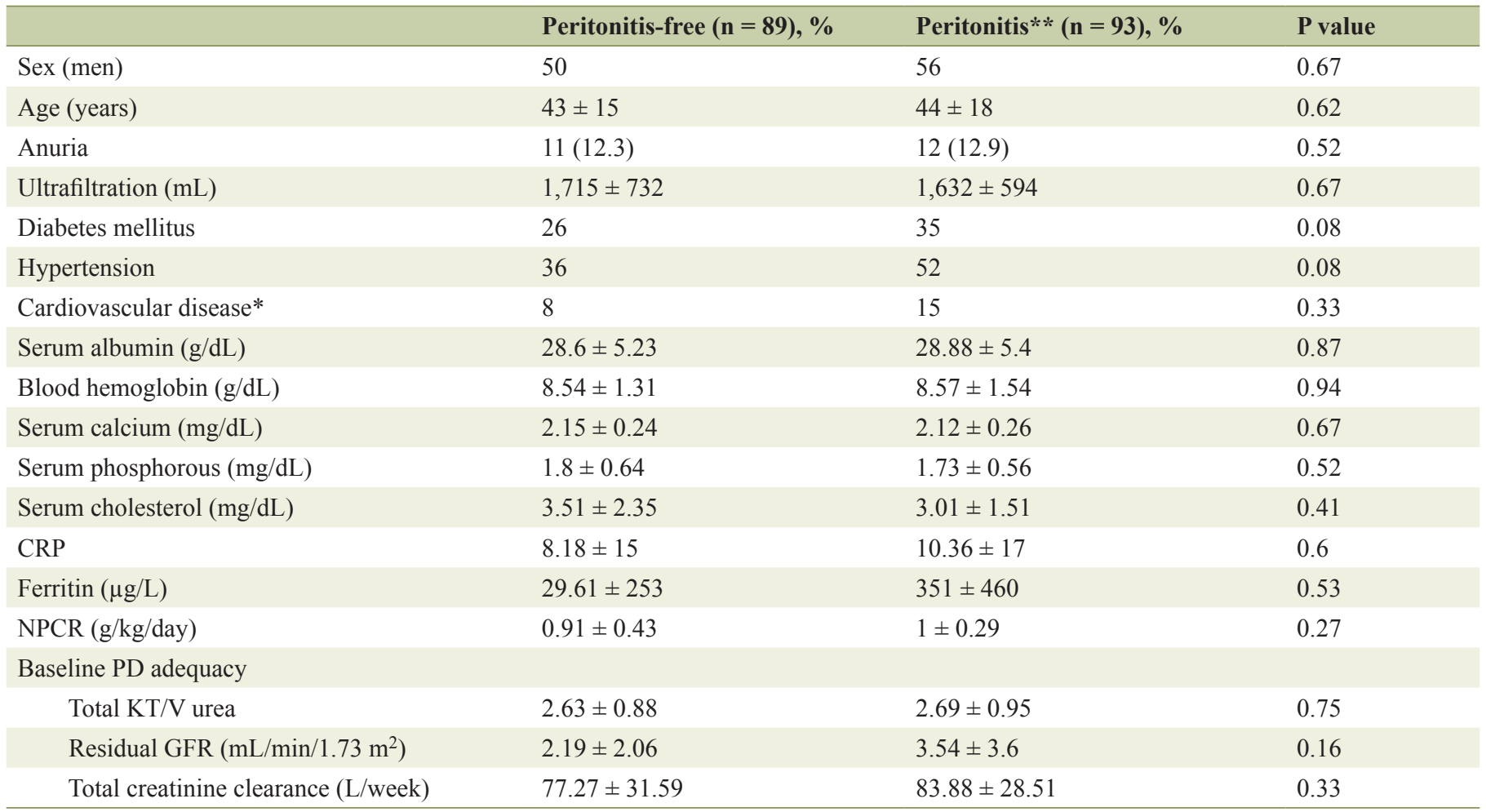

Values are expressed as mean $\pm \mathrm{SD}$, or number (\%). GFR: glomerular filtration rate (estimated by the average of renal BUN and creatinine clearance). ${ }^{*}$ Defined as the presence of coronary artery disease, cerebrovascular disease or peripheral artery disease. ${ }^{* *} A t$ least one peritonitis. CRP: C-reactive protein.

unit, it decreased significantly in the 1990 s to one episode/24 patient-months $[6,7]$ due to the development of disconnect systems and twin bag system $[8,9]$.

According to the CAPD Registry of the National Institutes of Health's report in 2004, it decreases to 0.4 episodes/patientyear [10].

While those reports indicate that our incidence of PDrelated peritonitis is not unusual compared with other countries, the latest Japanese report showed an incidence of one episode/73.5 month-patient [11]. The experience of Japanese medical centers with large numbers of well-controlled PD patients may explain this discrepancy [12].

Our study reports a detailed characterization of the microbiology and sources of peritonitis in a representative Tunisian population.

Gram-positive organisms were the main common agents (27.92\%), Gram-negative organisms caused $23.9 \%$ of peritonitis, fungi caused $6.5 \%$ of peritonitis, and no case of $M$. tuber- culosis has been reported.

Culture-negative peritonitis was seen in $36.55 \%$ of episodes. This rate exceeds the $20 \%$ limit set by the ISPD recommendations [13].

The proportion of negative culture reported in our study $(36.55 \%)$ is considered to be a high rate. This appears to be similar to two reports with an average of $36.9 \%$ and $31.8 \%$ in Korea and Tokai areas.

Our results can be compared to other reports summarized in Table 7 [1, 12, 14-17].

In the present study, we analyzed the data of CAPD and APD patients complicated by peritonitis and found that in the peritonitis-free group, diabetes, hypertension, and cardiovascular disease were less prevalent. Levels of ultrafiltration, CRP and ferritin were higher also in the peritonitis group.

From the current literature, many risk factors have been identified to predict peritonitis, including old age, race, obesity, diabetes, hypoalbuminemia, composition of dialysis solu-

Table 6. Cox Regression Model for Peritonitis Risk Factors

\begin{tabular}{llll} 
& \multicolumn{3}{c}{ Multivariate analysis } \\
\cline { 2 - 4 } & HR & $\mathbf{9 5} \%$ CI & P \\
\hline Residual GFR $\left(\mathrm{mL} / \mathrm{min} / 1.73 \mathrm{~m}^{2}\right)$ & 1.17 & $0.97-1.42$ & 0.085 \\
Diabetes mellitus & 0.77 & $0.27-2.15$ & 0.61 \\
Hypertension & 1.37 & $0.55-3.43$ & 0.49 \\
\hline
\end{tabular}




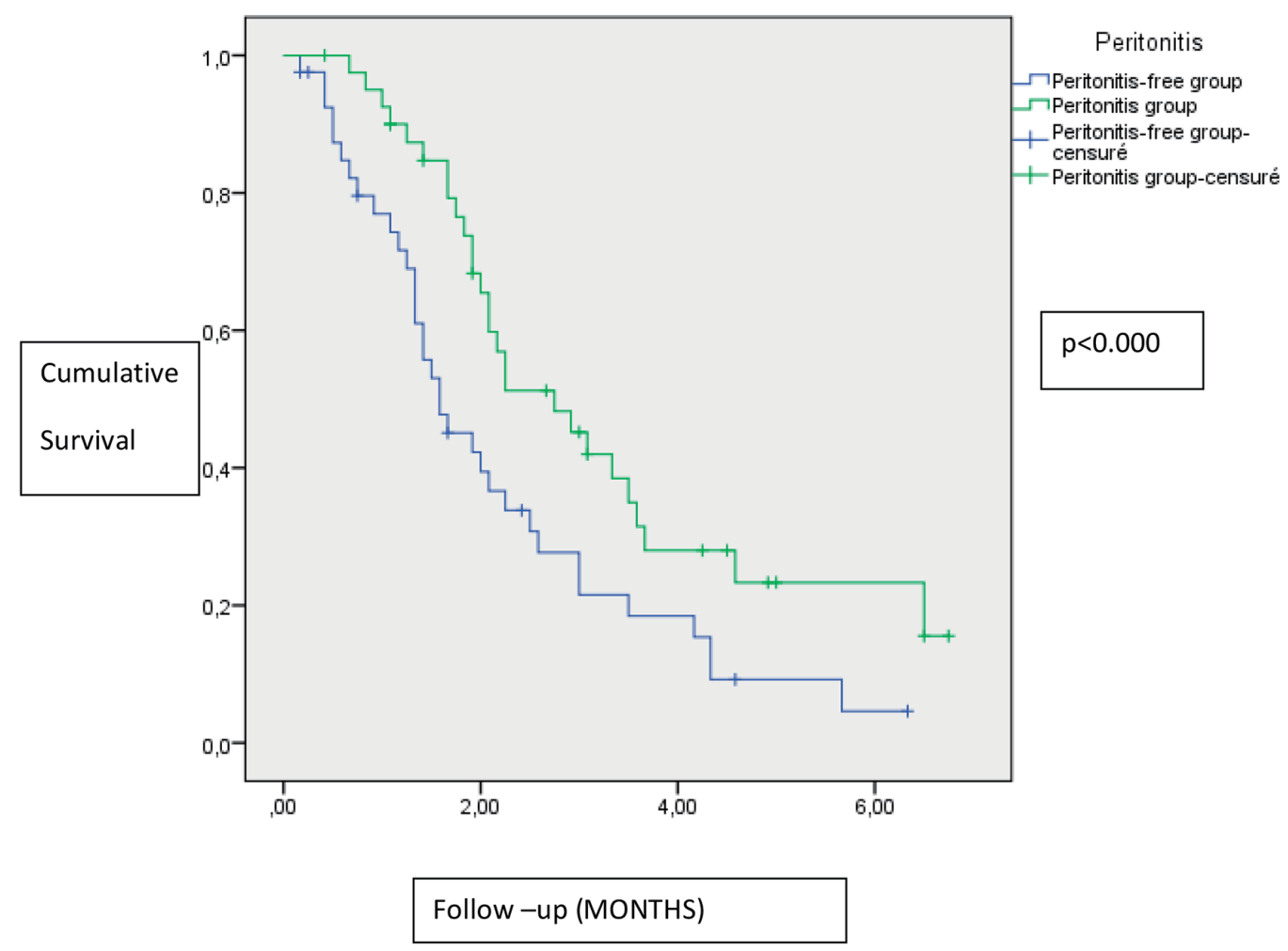

Figure 1. Impact of peritonitis on residual renal function.

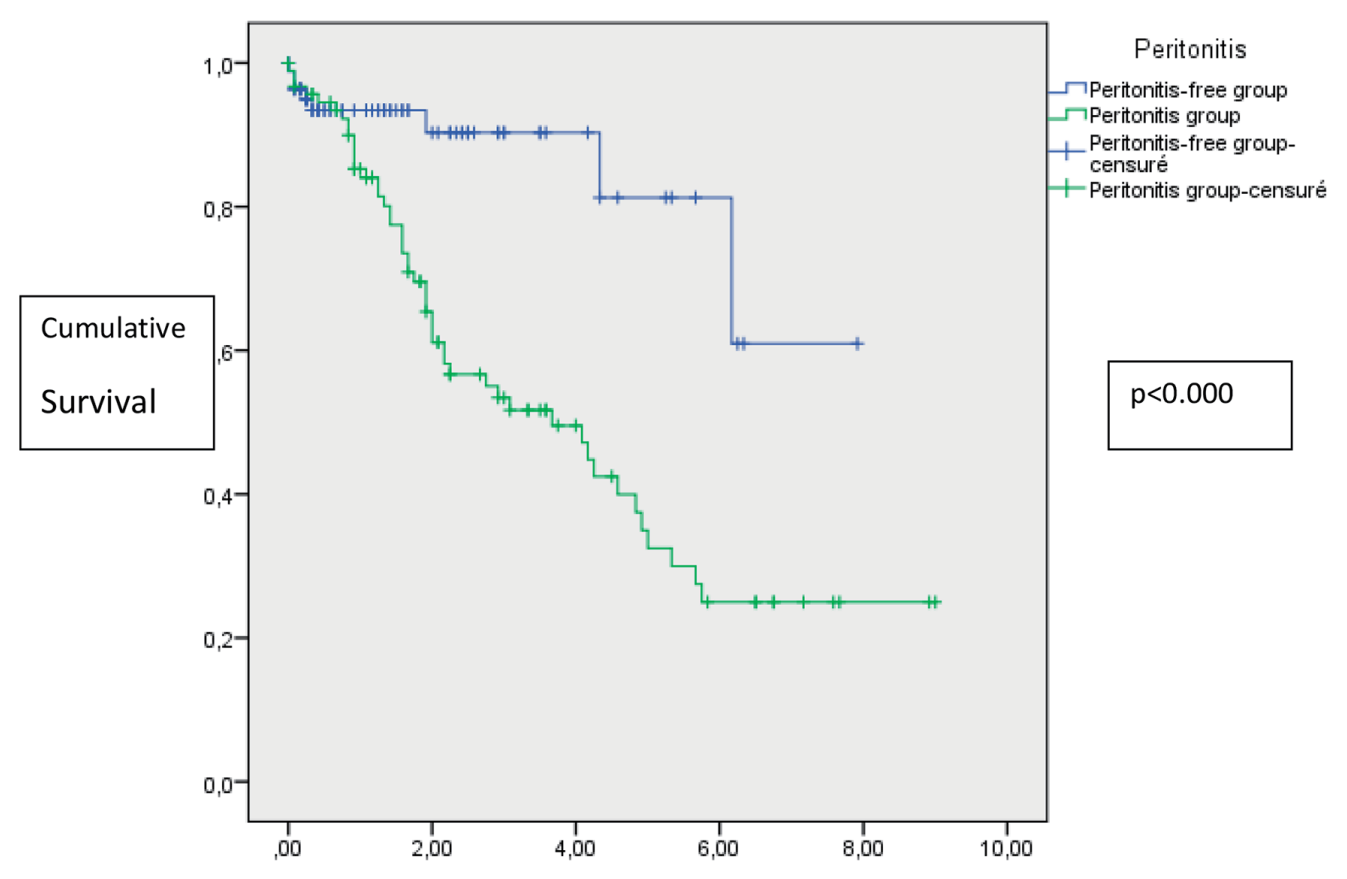

Follow -up (MONTHS)

Figure 2. Impact of peritonitis on technique survival. 


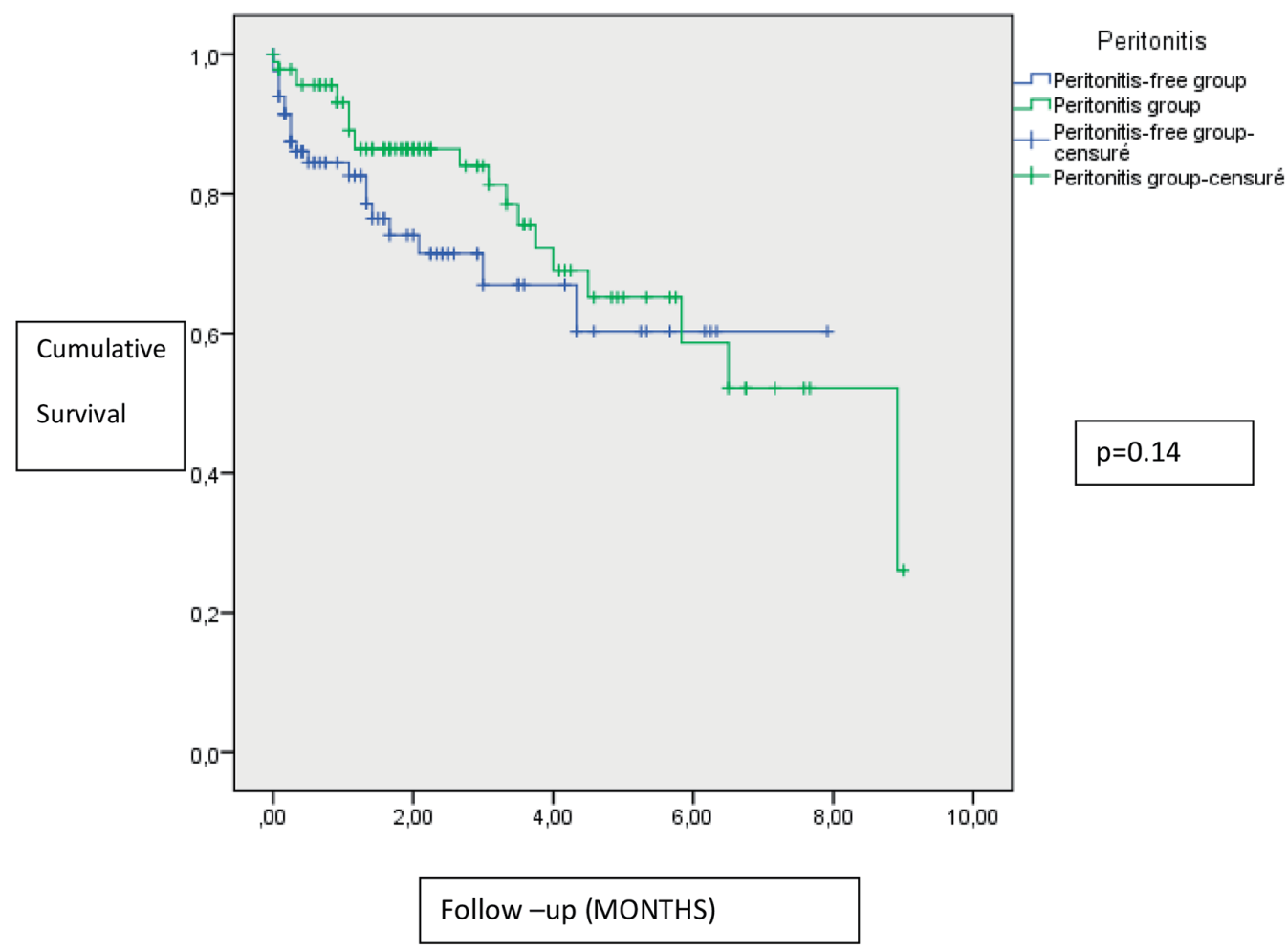

Figure 3. Impact of peritonitis on patient survival.

tions, improper bag exchange technique, lack of residual renal function, climate-related factors, geographical variability, local application of mupirocin at the catheter exit site, as well as transfer from HD and mode of PD [18, 19]. Whereas some of these factors are inconsistent in different reports.

In a PD Japanese study, 561 PD subjects were followed up, the risk factors advanced by the study were age $>65$ years and the female sex [12]. In this last study, the majority of patients were on CAPD.

The use of nasal mupiricin was associated with significantly reduced rates of peritonitis per person-month in Scotland [1]. In our study, the use of nasal mupiricin was prescribed only if the patient is bearing the Staphylococcus.

A recent observational study from Hong Kong found that CAPD patients with diabetes were at particularly high risk for peritonitis [20]. AGE products produced in diabetic condition were also found to be detrimental to phagocytic activity of peritoneal macrophages [21]. The difference between diabetic and non-diabetic persons was not significant but diabetes was less prevalent in the peritonitis-free group.

Peritonitis remains to be the most common cause of PD failure, with a significant impact on patient morbidity despite the important reduction of its incidence observed over the past years [3].

In our study, peritonitis was significantly associated with technique failure and dropout from PD, but not with RRF and patient survival.

Single episodes of peritonitis had no significant effect on peritoneal membrane function and ultrafiltration capacity.
Whereas, recurrent peritonitis might increase membrane permeability and reduce ultrafiltration independently of the causative organisms $[22,23]$. Peritonitis was the cause of technique failure in $42.6 \%$ of adult PD patients in a nationwide study in Scotland and $41.7 \%$ in London $[1,2]$. In the Tokai area of Japan, the most common reason for withdrawal PD was PD-related peritonitis $(20.7 \%)$, as in other countries. The major reason for transfer to HD from 2005 to 2007, in this Japanese study, was peritonitis $(26.7 \%)$, followed by dialysis failure $(21.3 \%)$ [12]. In Taiwanese CAPD patients, among 92 patients switching to HD, 42 patients $(45.7 \%)$ were attributed to peritonitis, followed by operational problems (in $18.5 \%$ of cases). In this study, the high peritonitis rate was associated with an increase in the risk of technique failure compared with low peritonitis rate [3].

In our study, there was no difference of patient survival between peritonitis group and peritonitis-free group.

Contradictory to other studies, peritonitis in our study did not influence patient survival. In the Taiwanese study, peritonitis contributed to a definite risk of mortality and resulted in death in $4.2 \%$ of the episodes. The high peritonitis rate was associated with an increase in the risk of patient mortality compared with low peritonitis rate [3].

Furthermore, in a Spanish retrospective analysis of a multicenter registry conducted from January 1993 to December 2005 including 1,515 patients, it was confirmed that lower long-term survival was associated with peritonitis with (hazard ratio 2.01, $\mathrm{P}<0.001)$. The hazard ratio differed according to the causal agent and was 1.73, 2.43 and 5.71 (for Gram- 


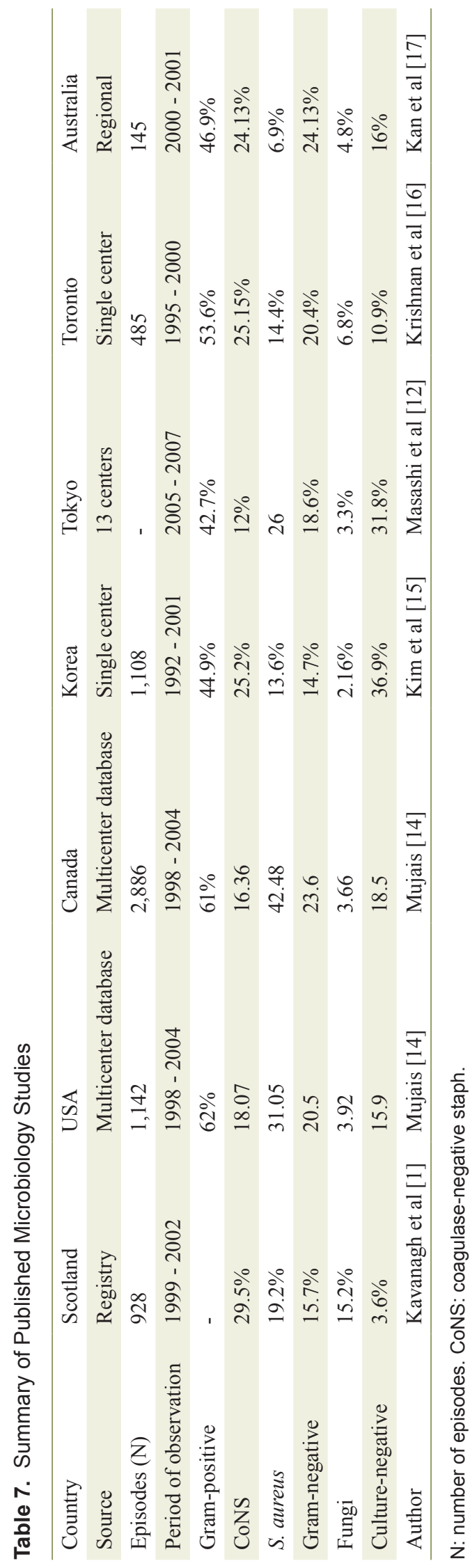

positive and Gram-negative bacteria and fungi), respectively.

This discrepancy with our results can be explained probably by the size of our population, because most of the studies that found an association with mortality were very broad and included at least 390 patients.

\section{Conclusion}

In summary, we confirm PD-associated peritonitis as a leading cause of technique failure but not mortality.

Proposed preventive strategies for peritonitis include proper technical training, patient selection, different PD systems (APD vs. CAPD), prophylactic antibiotics prior to catheter implantation, Y set and double-bag systems and prophylactic antibiotics to the exit site. The respect of these rules leads to a decrease in the proportion of peritonitis episodes.

\section{References}

1. Kavanagh D, Prescott GJ, Mactier RA. Peritoneal dialysis-associated peritonitis in Scotland (1999-2002). Nephrol Dial Transplant. 2004;19(10):2584-2591.

2. Davenport A. Peritonitis remains the major clinical complication of peritoneal dialysis: the London, UK, peritonitis audit 2002-2003. Perit Dial Int. 2009;29(3):297-302.

3. Hsieh YP, Chang CC, Wang SC, Wen YK, Chiu PF, Yang Y. Predictors for and impact of high peritonitis rate in Taiwanese continuous ambulatory peritoneal dialysis patients. Int Urol Nephrol. 2015;47(1):183-189.

4. Digenis GE, Abraham G, Savin E, Blake P, Dombros N, Sombolos K, Vas S, et al. Peritonitis-related deaths in continuous ambulatory peritoneal dialysis (CAPD) patients. Perit Dial Int. 1990;10(1):45-47.

5. Association R. Treatment of patients with renal failure: recommended standards and audit measures. London: Renal Association and Royal College of Physicians Palliative care for people with end stage renal disease. 2003;219.

6. Lee GS, Woo KT. Infection in continuous ambulatory peritoneal dialysis (CAPD): aetiology, complications and risk factors. Ann Acad Med Singapore. 1992;21(3):354-360.

7. Tielens E, Nube MJ, de Vet JA, van Limbeek J, Hofman $\mathrm{X}$, Steffens A, van Geelen JA. Major reduction of CAPD peritonitis after the introduction of the twin-bag system. Nephrol Dial Transplant. 1993;8(11):1237-1243.

8. Burkart JM, Hylander B, Durnell-Figel T, Roberts D. Comparison of peritonitis rates during long-term use of standard spike versus Ultraset in continuous ambulatory peritoneal dialysis (CAPD). Perit Dial Int. 1990;10(1):4143.

9. Daly CD, Campbell MK, MacLeod AM, Cody DJ, Vale LD, Grant AM, Donaldson C, et al. Do the Y-set and double-bag systems reduce the incidence of CAPD peritonitis? A systematic review of randomized controlled trials. Nephrol Dial Transplant. 2001;16(2):341-347.

10. Woodrow G, Turney JH, Brownjohn AM. Technique failure in peritoneal dialysis and its impact on patient sur- 
vival. Perit Dial Int. 1997;17(4):360-364.

11. Kawaguchi Y. Various obstacles to peritoneal dialysis development in Japan: too much money? Too much fear? Peritoneal Dialysis International. 2007;27(Supplement 2):S56-S58.

12. Mizuno M, Ito Y, Tanaka A, Suzuki Y, Hiramatsu H, Watanabe M, Tsuruta Y, et al. Peritonitis is still an important factor for withdrawal from peritoneal dialysis therapy in the Tokai area of Japan. Clin Exp Nephrol. 2011;15(5):727-737.

13. Li PK, Szeto CC, Piraino B, Bernardini J, Figueiredo AE, Gupta A, Johnson DW, et al. Peritoneal dialysis-related infections recommendations: 2010 update. Perit Dial Int. 2010;30(4):393-423.

14. Mujais S. Microbiology and outcomes of peritonitis in North America. Kidney Int Suppl. 2006;103:S55-62.

15. Kim DK, Yoo TH, Ryu DR, Xu ZG, Kim HJ, Choi KH, Lee HY, et al. Changes in causative organisms and their antimicrobial susceptibilities in CAPD peritonitis: a single center's experience over one decade. Perit Dial Int. 2004;24(5):424-432.

16. Krishnan M, Thodis E, Ikonomopoulos D, Vidgen E, Chu M, Bargman JM, Vas SI, et al. Predictors of outcome following bacterial peritonitis in peritoneal dialysis. Perit
Dial Int. 2002;22(5):573-581.

17. Kan GW, Thomas MA, Heath CH. A 12-month review of peritoneal dialysis-related peritonitis in Western Australia: is empiric vancomycin still indicated for some patients? Perit Dial Int. 2003;23(5):465-468.

18. Dong J, Chen Y. Impact of the bag exchange procedure on risk of peritonitis. Perit Dial Int. 2010;30(4):440-447.

19. Kim MJ, Song JH, Park YJ, Kim GA, Lee SW. The influence of seasonal factors on the incidence of peritonitis in continuous ambulatory peritoneal dialysis in the temperate zone. Adv Perit Dial. 2000;16:243-247.

20. Chow KM, Szeto CC, Leung CB, Kwan BC, Law MC, Li PK. A risk analysis of continuous ambulatory peritoneal dialysis-related peritonitis. Perit Dial Int. 2005;25(4):374379.

21. Liu BF, Miyata S, Kojima H, Uriuhara A, Kusunoki H, Suzuki K, Kasuga M. Low phagocytic activity of resident peritoneal macrophages in diabetic mice: relevance to the formation of advanced glycation end products. Diabetes. 1999;48(10):2074-2082.

22. Brown EA. Peritonitis: limiting the damage. Nephrol Dial Transplant. 2005;20(8):1539-1541.

23. Bailie GR. Therapeutic dilemmas in the management of peritonitis. Perit Dial Int. 2005;25(2):152-156. 